\title{
Use of Multi-Rotor Unmanned Aerial Vehicles for Radioactive Source Search
}

\author{
Bai Li ${ }^{1}{ }^{10}$, Yi Zhu ${ }^{1,5}$, Zhanyong Wang ${ }^{1,2, *(1)}$, Chao Li $^{1}$, Zhong-Ren Peng ${ }^{1,3,4, *}$ and Lixin Ge ${ }^{6}$ \\ 1 Center for Intelligent Transportation Systems and Unmanned Aerial Systems Applications, State Key \\ Laboratory of Ocean Engineering, School of Naval Architecture, Ocean \& Civil Engineering, Shanghai Jiao \\ Tong University, Shanghai 200240, China; bai_li@sjtu.edu.cn (B.L.); zhu.yi@mail.shufe.edu.cn (Y.Z.); \\ lichao_rusty@sjtu.edu.cn (C.L.) \\ 2 School of Engineering, Sun Yat-sen University, Guangzhou 510006, China \\ 3 China Institute for Urban Governance, Shanghai Jiao Tong University, Shanghai 200240, China \\ 4 International Center for Adaptation Planning and Design, School of Landscape Architecture and Planning, \\ College of Design, Construction, and Planning, University of Florida, P.O. Box 115706, \\ Gainesville, FL 32611-5706, USA \\ 5 School of Public Economics and Administration, Shanghai University of Finance and Economics, \\ Shanghai 200240, China \\ 6 Shanghai Institute for Supervision over Radio-environment, Shanghai 200065, China; gelix68@126.com \\ * Correspondence: wangzy1026@163.com (Z.W.); zrpeng@sjtu.edu.cn (Z.-R.P.)
}

Received: 21 March 2018; Accepted: 6 May 2018; Published: 9 May 2018

\begin{abstract}
In recent years, many radioactive sources have been lost or stolen during use or transportation. When the radioactive source is lost or stolen, it is challenging but imperative to quickly locate the source before it causes damage. Nowadays, source search based on fixed-wing unmanned aerial vehicles (UAVs) can significantly improve search efficiency, but this approach has higher requirements for the activity of the uncontrolled radioactive source and the take-off sites. The aim of this study was to design and demonstrate a platform that uses low-cost multi-rotor UAVs to automatically and efficiently search for uncontrolled radioactive sources even with lower activity. The hardware of this platform consists of a multi-rotor UAV, radiation detection sensor, main control module, gimbal and camera, and ground control station. In the search process, the ground control station and UAV communicate wirelessly in real time. To accommodate different search scenarios, the study proposed three search algorithms with a theoretical comparison. Then, field experiments based on the traversal search algorithm showed that the search system based on multi-rotor UAVs could effectively and accurately conduct contour mapping of a region and locate the radioactive source with an error of $0.32 \mathrm{~m}$. The platform and algorithms enable accurate and efficient searching of radioactive sources, providing an innovative demonstration of future environmental risk assessment and management.
\end{abstract}

Keywords: uncontrolled radioactive sources; source search algorithm; multi-rotor UAV; contour mapping

\section{Introduction}

Over the past ten years, certain radioactive sources have been accidentally lost and stolen worldwide [1,2]. Three incidents have taken place in Shanghai, and similar accidents have also occurred in neighboring provinces and cities [3]. In the actual search and disposal process, in addition to a small number of conditions that allow use of search vehicles, most cases are still forced to apply the large-crowd strategy, which mobilizes a large number of people with portable instruments for a wire-type search. Practice has shown that this method is highly inefficient and might be harmful to 
operators. Therefore, the search for uncontrolled radioactive sources (i.e., lost or stolen radioactive sources) requires more efficient and safer methods.

Contour mapping and source search as an attractive research topic has drawn much attention in a radioactive field [4-6]. In a typical search for lost or stolen radioactive sources, five main strategies are used to conduct search activities. Radiation detection devices are carried by emergency personnel [7], vehicles [8], manned aircrafts [1], or unmanned aerial vehicles (UAVs) to search uncontrolled radioactive sources in a certain region. Apart from these approaches, a distributed sensor network deployed in a region can also infer the radioactive source location based on the fusion information from each sensor $[9,10]$. Nemzek et al. compared the performance of a distributed sensor network and single sensor in the radioactive source search, and found that an increase in the number of sensors led to a higher signal-to-noise ratio, even if the radioactive source was in motion [11]. Brennan et al. demonstrated that compared with portable detection devices, distributed sensing networks were easier to rapidly deploy and were insensitive to the speed of the carrier during field monitoring [9]. Sundaresan et al. proposed a distributed sensor network that contained many sensor nodes. They found that each sensor made a local decision over a period of time, while the global decision was made by the fusion center. These researchers also obtained the optical sensor thresholds using the normal copula function based on the copulas theory [12].

However, methods for searching uncontrolled radiation sources in a region based on persons, vehicles, and manned aircrafts all require operators to enter the region, which easily exposes operators to radiation from the lost sources. In addition, manual search is usually limited by walking speed and radiation protection standards, and hence cannot meet the demands of rapid and accurate search for uncontrolled radioactive sources. The search method in which a vehicle carries a large-scale radiation detection device has higher requirements for road typology and accessibility in the region. Thus, it cannot be well adapted to complex terrains, such as mountainous and urban regions. Although the distributed sensor network can address the defects of the above two methods to a certain extent, it is necessary to disassemble and redeploy the sensor network in a radioactive source search in different regions, which means that flexible switching between different search regions is impossible. In contrast, the high mobility makes UAVs easy to deploy and less restricted by geographical conditions [13]. Therefore, the search based on UAVs can address the shortcomings of other methods mentioned above and has unique advantages in the search for uncontrolled radioactive sources.

In the field of radiation detection, small radiation monitoring units that are suitable for UAVs and also contain GPS wireless transmission modules have been in development for a period of time. These units supply the technical support and research foundation for the application of UAVs in radiation detection. Based on simulation scenarios of nuclear radiation, Václav Smídl et al. designed a new method for automatic tracking and detection of pollutant emission by using a UAV equipped with a portable sensor [14]. Their simulation results showed significant improvement compared with stationary monitoring networks. Pöllänen et al. conducted a radiation detection experiment using a small UAV with a specially designed sampling unit [15]. Results showed that the ${ }^{137} \mathrm{Cs}$ radioactive source could be detected at a height of $50 \mathrm{~m}$ and flight speed of $70 \mathrm{~km} / \mathrm{h}$. In the Fukushima nuclear accident in Japan, the Japanese government used drones to perform nuclear radiation surveys in order to better understand the distribution and spread of nuclear radiation. In the majority of the existing scenarios, the searchers and operators chose fixed-wing UAVs due to their low cost and high flexibility. However, because the fixed-wing UAVs cannot hover in the air, one common choice is to allow UAVs to fly along a circular path around the source [16]. Nowadays, the development of a UAV flight control system has not only strengthened the control of a single UAV but also realized control of a coordinated flight of multiple UAVs [17-19]. Han et al. developed a low-cost UAV platform and then used multiple low-cost UAVs to detect a radiation diffusion profile. The UAV flight scheme also varied with different radiation scenes. Both simulations and experiments have proved the feasibility of three-dimensional radiation detection based on multiple UAVs [20]. In subsequent research, Han et al. conducted the search for an uncontrolled radioactive source and also performed contour mapping of a radioactive 
field using multiple low-cost UAVs. There were four scenarios presented in the study to illustrate the feasibility of the search and mapping algorithms [21].

Most of these studies used fixed-wing UAVs in the search for a radioactive source with high activity, but in most cases the lost or stolen radioactive sources had lower activity (classified as IV or below). This scenario often displays a variation in the dose rate that cannot be detected at more than several meters from the radioactive source. At this point, traditional methods of radiation source search encounter difficulties. However, because of the absence of stall speed restrictions, multi-rotor UAVs are able to search for the uncontrolled radioactive source at a low speed and a low altitude, which becomes highly effective for low-activity radioactive sources. Once the source is located, the UAV can hover over the potential radioactive source for crawling or other follow-up operations. The motivation of this study is to demonstrate a search platform based on a low-cost, multi-rotor UAV that can automatically and efficiently search for an uncontrolled radioactive source. The study presents the design of hardware and software for the search system. Three different algorithms for radioactive source search are proposed and theoretically compared. A field experiment is then performed to demonstrate the reliability of the search system and the effectiveness of one of the algorithm.

\section{Materials and Methods}

Radioactive source detection based on multi-rotor UAVs in an unknown region faces many challenges. First, it is important to select a suitable lightweight, high-precision nuclear radiation detection sensor that can be mounted on the UAVs. Second, it is also critical to design or pick an appropriate search algorithm based on the dose rate data. In this study, the hardware architecture of the radioactive source search system consists of a hex-rotor UAV with a gimbal and camera, a radiation detection sensor, an embedded Linux development board and a ground control station (GCS). Three algorithms are adopted in this search system to achieve flexible radioactive source search execution under different situations.

\subsection{Hardware Architecture}

Figure 1 shows the hardware architecture of the search system, which consists of three main components: UAV side, ground control station, and remote controller. The main control module is the center of the UAV side. Other components, including the flight control module, radiation detection sensor, transmission radio, and debugger, are connected with the main control module through a universal asynchronous receiver/transmitter (UART). The central control module reads the UAV status and issues control commands via two-way information communication with the flight control module. The radiation detection sensor sends the radiation dose rate monitoring information to the main control module at a $1 \mathrm{~Hz}$ frequency through the UART. The main control module facilitates far and near two-way wireless communication with the ground control station using a $433 \mathrm{MHz}$ wireless link and Bluetooth via the transmission radio and the debugger. Similarly, transmission of the UAV status and radiation dose rate information to the ground control station and reception of control commands from the ground control station are also accomplished in this manner. A Zenmuse $\mathrm{Z} 3$ gimbal and camera $(\mathrm{Z3})$ is directly connected to the flight control module via a wired link. The flight control module compresses the video information received from the $\mathrm{Z} 3$ and sends it directly to the remote controller via the $2.4 \mathrm{GHz}$ wireless link. The channel also transmits control commands to the UAV via the remote controller. The ground control station is centered on the PC; the transmission radio and the debugger are connected to the PC via the UART. The debugger or transmission radio immediately forwards the information to the PC after receiving it from the UAV. The software running on the PC decodes the information, obtains the status of the UAV and the radiation dose rate data, and displays the data on the software interface. At the same time, the control commands for UAV-side devices are also sent to the UAV via the debugger or transmission radio. Additionally, the debugger is also used to transport the initial information required by the source search algorithm (the length and width of the search site, the desired search height, and flight speed, etc.) to the UAV before UAV 
takeoff. The telecontroller of the remote controller is connected to the mobile device via USB, and the mobile device runs the DJI GO App. When the telecontroller receives the video stream returned by the UAV through the $2.4 \mathrm{GHz}$ wireless link, the video stream is sent directly to the mobile device through the USB connection, and the real-time video is decoded and displayed by the mobile device. Control commands for the UAV from the telecontroller are also sent to the UAV via the channel. It should be noted that the control commands of the remote controller can only control the UAV attitude and the $\mathrm{Z3}$, and the control commands of the ground control station can control the UAV attitude and Z3 and the radiation detection sensor. Figure 2 shows the images of the radioactive source search system, and each item is described separately.

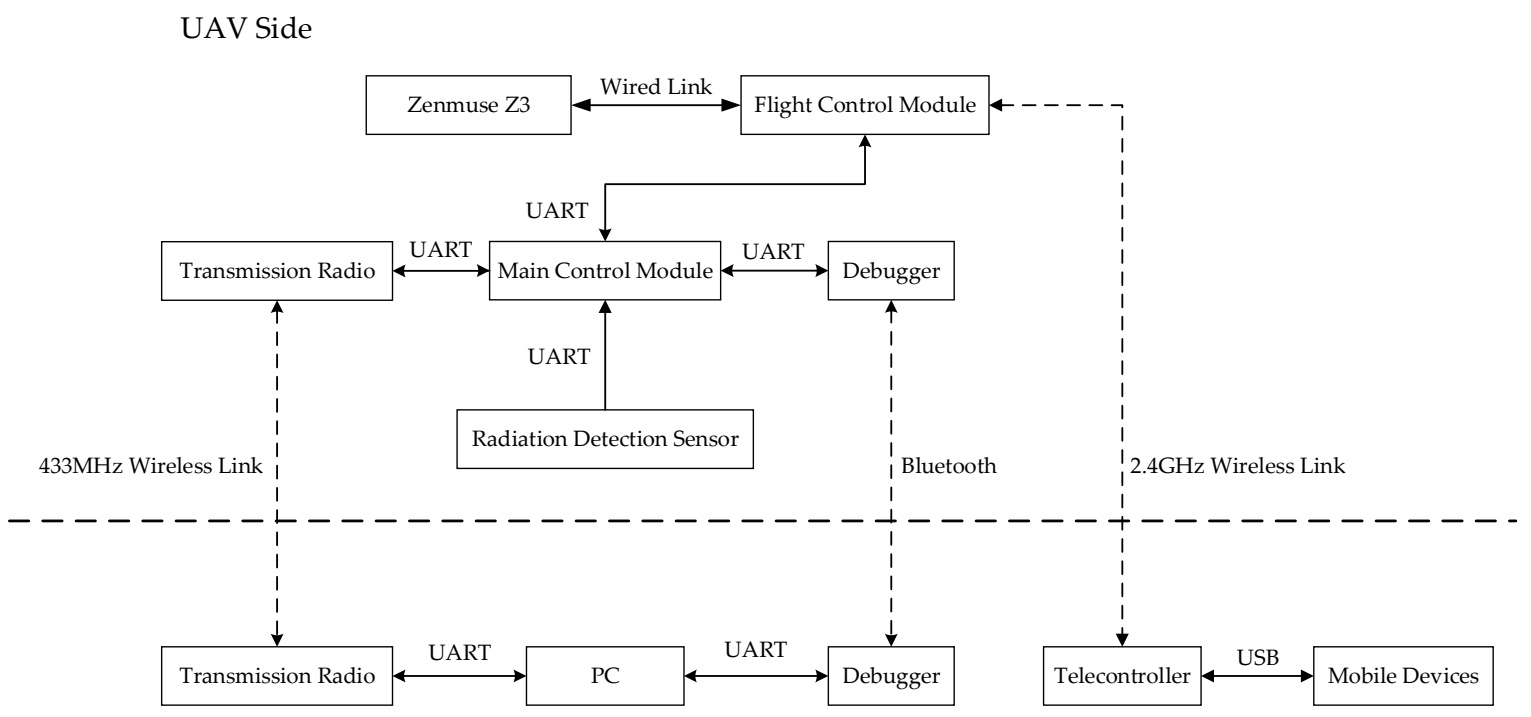

Ground Control Station

Remote Controller

Figure 1. Hardware architecture of the source search system.

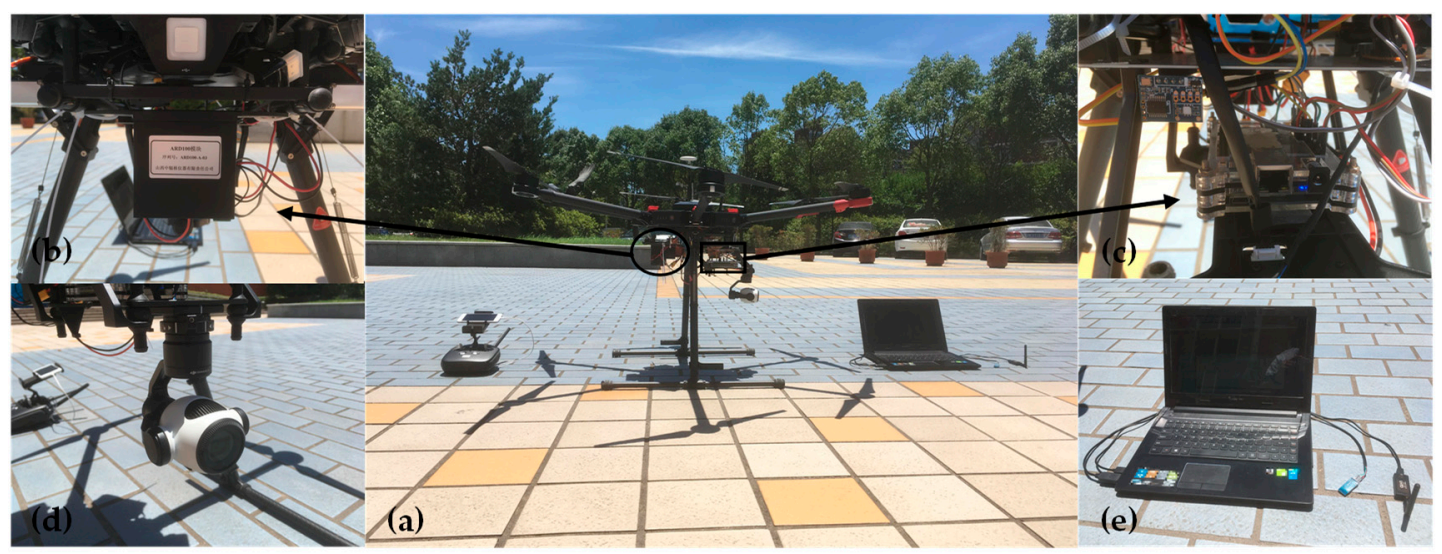

Figure 2. Sketch map of the search system. (a) DJI Matrice 600 UAV platform; (b) radiation detection sensor; (c) main control module; (d) Zenmuse Z3 gimbal and camera; (e) ground control station.

\subsubsection{DJI Matrice 600 UAV Platform}

The DJI Matrice 600 hexacopter (M600) is a hex-rotor UAV platform released by DJI (14th Floor, West Wing, Skyworth Semiconductor Design Building, No.18 Gaoxin South 4th Ave, Nanshan District, Shenzhen, China, 518057) in 2016. For ease of transportation and installation, the UAV adopts a folding structure design, i.e., the propellers, arms, and GPS holder can be folded. After the UAV is fully 
extended, the size is $1668 \mathrm{~mm} \times 1518 \mathrm{~mm} \times 727 \mathrm{~mm}$. The max ascent speed, max descent speed, max speed, and max wind resistance are $5 \mathrm{~m} / \mathrm{s}, 3 \mathrm{~m} / \mathrm{s}, 18 \mathrm{~m} / \mathrm{s}$, and $8 \mathrm{~m} / \mathrm{s}$, respectively. The max payload of the UAV reaches $5.5 \mathrm{~kg}$. With the capacity for six $5700 \mathrm{mAh}$ TB48S batteries, in the full load case, the effective flight time of the UAV can reach $18 \mathrm{~min}$. In our study, if equipped with all the devices and sensors, the lifetime of the M600 can reach $30 \mathrm{~min}$, which is sufficient to meet the requirements of most uncontrolled radiation source search tasks. To facilitate the development of customized applications for professional users, the M600 offers an application programming interface (API) and two $18 \mathrm{~V}$ power interfaces. Users can install a development board on the UAV to achieve two-way communication between the development board and flight control module through the API. Based on the onboard software development kit (OnboardSDK) and mobile software development kit (MobileSDK) supplied by DJI, users can implement an automatic operation of the UAV system by writing program runs in the development boards or mobile devices.

\subsubsection{Radiation Detection Sensor}

In the case of a search for stolen or lost uncontrolled radioactive sources, the characteristics of the type and activity of the uncontrolled radioactive source might vary greatly, and thus the dose rates might significantly differ. Therefore, the radiation detection sensor must have both a broad range and high sensitivity. In this study, an ARD100 radiation detection sensor developed by Shanxi Zhongfu Nuclear Instruments Co., Ltd (No.420, Changzhi Road, Taiyuan City, Shanxi Province, China, 030006). is used as the airborne sensor. Table 1 shows the specifications of the sensor in detail.

The sensor uses a dual detector scheme. One detector measures the low range, approximately $0.1 \mu \mathrm{Sv} / \mathrm{h}$ to $0.6 \mathrm{mSv} / \mathrm{h}$, and the other detector measures the high range of approximately $0.6 \mathrm{mSv} / \mathrm{h}$ to $1 \mathrm{~Sv} / \mathrm{h}$. The dual detector scheme implements a dose rate measurement range of the sensor of $1 \mu \mathrm{Sv} / \mathrm{h}$ to $500 \mathrm{mSv} / \mathrm{h}$, and the detection sensitivity of the low range reaches $100 \mathrm{cps} / \mu \mathrm{Sv} / \mathrm{h}$. The low range detector uses a silicon photodetector $+\mathrm{CsI}(\mathrm{Tl})$ scintillator scheme. The scintillator volume is $15 \mathrm{~mm} \times 15 \mathrm{~mm} \times 15 \mathrm{~mm}$. The silicon photodetector uses NDL-11, with a sensitive area of $6 \mathrm{~mm} \times$ $6 \mathrm{~mm}$, a detection wavelength of 300-800 (nm), and wavelength efficiency $(420 \mathrm{~nm})>42 \%$. The high range detector uses a silicon PIN semiconductor detector with a detection area of $7.54 \mathrm{~mm}^{2}$.

Table 1. Radiation detection sensor specifications.

\begin{tabular}{cc}
\hline Properties & Value \\
\hline Dose rate range & $0.1 \mu \mathrm{Sv} / \mathrm{h}-1 \mathrm{~Sv} / \mathrm{h}$ \\
Energy range & $50 \mathrm{keV}-1.5 \mathrm{MeV}$ \\
Accuracy & $20 \%$ \\
Relative angular response & $\geq 0.7 \mathrm{in} \pm 90^{\circ}$ \\
Response time & $\leq 1 \mathrm{~s}$ \\
High range sensitivity & $\geq 0.03 \mathrm{cps} / \mu \mathrm{Sv} / \mathrm{h}$ \\
Low range sensitivity & $\geq 100 \mathrm{cps} / \mu \mathrm{Sv} / \mathrm{h}$ \\
Dimensions & $155 \mathrm{~mm} \times 84 \mathrm{~mm} \times 70 \mathrm{~mm}$ \\
Weight & $240 \mathrm{~g}$ \\
Environment & $\leq 95 \% ;-25^{\circ} \mathrm{C}$ to $+55^{\circ} \mathrm{C}$ \\
\hline
\end{tabular}

\subsubsection{Main Control Module}

The central processor unit of the main control module is the BeagleBone Black development board produced by the TI Company (12500 TI Boulevard Dallas, Texas 75243, USA). The development board adopts the TI AM335× $1 \mathrm{GHz}$ ARM Cortex-A8 processor and has 512 MB DDR3 RAM. The 4-GB 8-bit eMMC onboard flash storage offers ample space for storing programs and data. The onboard NEON floating-point accelerator accelerates the floating point data processing speed, while the $2 \times$ PRU 32-bit microcontrollers make up for the shortcomings of Linux systems, which cannot handle real-time events. Regarding interfaces, the board contains 65 general purpose input/output interfaces (GPIOs), 8 PWMs and 4 timers, 7 analog inputs $(1.8 \mathrm{~V}), 4$ UARTs and 1 TX only, $2 \mathrm{I}^{2} \mathrm{C}$ ports, and 2 
SPI ports. This richness in the number of interfaces with a powerful central processor means that the development board can simultaneously read multiple sensor data, integrate these data, and finally realize two-way real-time communication between the UAV side and the GCS.

In this study, the dose rate information is obtained from the radiation detection sensor at a rate of once every second. The data are packaged with corresponding position information. Subsequently, the fused data are stored in the main chip and transmitted to the GCS simultaneously. Thus, the state of the UAV can be understood during the search process, and if any accidents happen to the UAV, the fused data can also be downloaded to the host computer for further analysis.

\subsubsection{Zenmuse Z3 Gimbal and Camera}

In conjunction with the Zenmuse Z3 gimbal and camera (Z3), emergency personnel is able to view the situation in real-time while the UAV searches for radiation sources. The Z3 offers $3.5 \times$ optical zoom and $2 \times$ digital zoom, and a maximum $7 \times$ zoom can be realized by combining the optical and digital zoom. The $\mathrm{Z} 3$ can transmit the video stream to the mobile devices in real time and store photos and videos on the SD card carried onboard. The most extended video transmission distance of the Z3 is $5 \mathrm{~km}$.

\subsubsection{Ground Control Station}

In order to monitor the search status and obtain the position information and corresponding dose rate information in real time during an automatic search for the uncontrolled radioactive source while maintaining control of the UAV, it is critical to realize data visualization during the entire process.

The ground station software, which is mainly used in human-computer interactions, runs on a PC and is written by Qt Creator. Prior to UAV takeoff, emergency personnel input the length and width of the search site, the expected cruising height, and the speed information to the software. The information is uploaded to the UAV side. After UAV takeoff, the software receives the longitude, latitude, height, and dose rate data from the UAV side. These data are displayed in the software interface in real time such that emergency personnel can understand the search process clearly.

\subsection{Software Architecture}

The search for a radioactive source in an unknown region relies on two main data sources: position information and dose rate information at different locations. This section introduces the software architecture of the radioactive source search system from the perspective of the source search algorithm.

With the position and the dose rate information at a particular position, a specific source search algorithm is needed to dictate the radioactive source search process. Different from typical fixed-wing UAVs, it is quite convenient for hex-rotor UAVs to hover and fly with low speed at super-low altitude. Based on this advantage, three source search algorithms were proposed and compared in this study. All of these algorithms simplify the source search problem to a two-dimensional situation by controlling the quadcopter to fly at a constant height.

The dose rate measured by a radiation detection sensor at the same distance from the radioactive source can be wildly different for radioactive sources of different types and with different activities. Moreover, a radiation detection sensor might also display a certain extent of measurement errors. Thus, to calculate the distance between the radioactive resource and the radiation detection sensor, the type and activity of the radioactive source is required first. Because of the measurement errors, the distance result retrieved from the dose rate measured by the sensor is inaccurate. This situation reduces the universality of source search algorithms and reduces the ability to search the uncontrolled radioactive source efficiently. In contrast, the algorithms proposed in this paper are all based on the concept of searching for the position with the highest level of dose rate in the region. This method improves the universality of the source search algorithms and increases the accuracy of the source search result. 


\subsubsection{Basic Assumptions}

A potential area that might contain a radioactive source is delineated before the source search process is performed by the UAV. To simplify the problem, we assume that the search area is a rectangle that stretches $P$ meters from east to west and Q meters from north to south. We also assume that the step size of the $x$-axis is M meter(s) and the step size of the $y$-axis is $\mathrm{N}$ meter(s) after area discretization, and that the airspeed throughout the flight is $S$ meter(s) per second. The specific discrete values of $M$ and $\mathrm{N}$ differ depending on the range of the search area. The two-dimensional rectangular Cartesian coordinate system is established at the beginning, and the original point is determined by the initial position of the UAV. The positive direction of the $x$-axis is the eastward direction, and the positive direction of the $y$-axis is the northward direction. The three algorithms introduced below both use the same assumptions.

\subsubsection{Traversal Search}

The algorithm block diagram is shown in Figure 3a. In a specific traversal search process, the initial position of the quadcopter is the southwest corner of the search area. The UAV first flies towards the east at a uniform airspeed of $S$ meters per second. Once the UAV reaches the point $(P, 0)$ in the coordinates, the UAV continues to fly towards the north at the same uniform speed until it reaches the point $(\mathrm{P}, \mathrm{N})$. Subsequently, the UAV flies back towards the west at the same uniform speed until it reaches the point $(0, N)$ and continues to fly towards the north at the same uniform airspeed to reach the point $(0,2 \mathrm{~N})$. At this moment, the UAV flies towards the east at the same airspeed until it reaches the point $(\mathrm{P}, 2 \mathrm{~N})$. Using such a circulation pattern, the UAV can search the entire area along an S-type path. Finally, the position with the maximum dose rate is calculated by the main control module, and the UAV hovers over this position to inform the operators that the most likely radioactive source has been found. At this time, the entire radioactive source search process is completed. Subsequently, the dose rate data can be exported for further analysis, e.g., an illustration of dose rate heat map of the entire area can be generated for further research. Since the implementing of the search algorithm needs to traverse the region, the time complexity of the algorithm is $O\left(n^{2}\right)$.

To save time and improve the efficiency of radioactive source search to the greatest extent, subsequent algorithms are not built based on traversal search but through observation of selected points in the area to determine the direction of the next step. In this manner, the UAV can approach the radioactive source in a step-by-step manner to complete the radioactive source search mission.

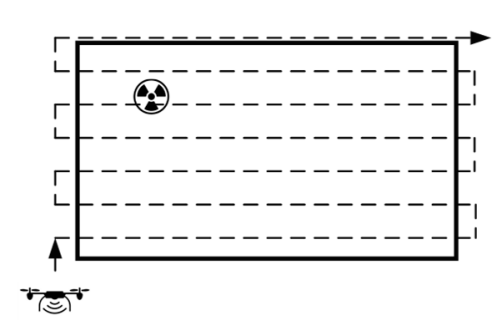

(a)

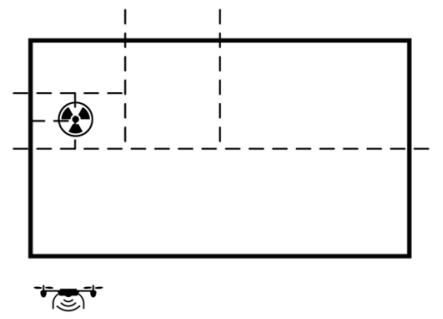

(b)

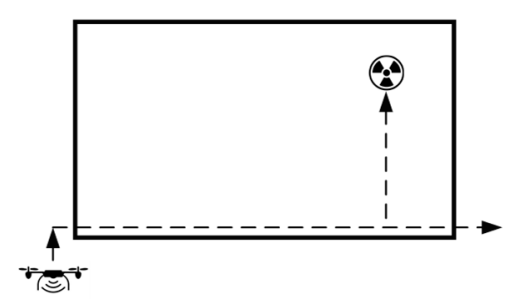

(c)

Figure 3. Diagram for each search algorithm. (a) Traversal search; (b) Binary search; (c) Successive approximation.

\subsubsection{Binary Search}

This algorithm is an extension of the binary search algorithm in data structures and is shown in Figure 3b. Taking the search between the southern boundary and the northern boundary as an example, in this scenario, the UAV flies along the northern boundary, the southern boundary, and the bisector between the northern and the southern boundary. The main control module calculates the mean dose rate of the three flights. The path with the lowest dose rate is discarded, and the two reserved paths become the new northern and the new southern boundary lines. The procedure 
mentioned above is performed over and over again until the distance between the two boundaries is less than a pre-defined distance. The search between the eastern boundary and the western boundary is also conducted in accordance with the above method. The two-dimensional binary search process can zoom down to a relatively small area that is likely to contain the radioactive source. The algorithm is applicable to the uncontrolled radioactive source with higher activity, so a significant dose rate can be detected within $1 / 4$ of the search area. Compared to the traversal search algorithm, this algorithm significantly reduces the time complexity by discarding half of the area each time to achieve $O(\log N)$.

\subsubsection{Successive Approximation}

Because the three-dimensional distribution of the dose rate of a radioactive source is a uniform sphere, the two-dimensional distribution of dose rate is a uniform circular shape. To further reduce the search time and improve the efficiency of the search procedure, combined with the theory that the perpendicular bisector of a line segment in a circle that belongs to the line that cuts the circle must pass through the center of the circle, a brand new search algorithm can be proposed as shown in Figure 3c. The UAV flies along the longer boundary of the detection area first. Once the flight is complete, the main control module selects the position with the highest dose rate of the path and controls the UAV to fly to this position. Subsequently, the UAV flies towards the internal area along the direction perpendicular to the initial flight. Once the flight is complete, the main control module selects the position with the highest dose rate of the entire path as the location of the radioactive source. However, the algorithm severely requires the uncontrolled radioactive source with higher activity than the binary algorithm, so the obvious change of dose rate can be detected on the boundary of the region. Compared with the first two algorithms, this algorithm reduces the time complexity to a minimum, reaching $O(1)$.

\subsection{Field Experiment}

Because the radioactive source has certain radioactivity, it is harmful to the environment and biological organisms. Therefore, radioactive sources are subject to highly strict supervision to prevent nuclear accidents. For the reasons mentioned above, researchers are not at liberty to perform radioactive source search experiments by themselves. In this study, the experiments on a simulated uncontrolled radioactive source search were conducted in collaboration with the Shanghai Station of Radiation Environment Supervision. To protect social production and personal safety, the experiment was conducted at the Shanghai municipal radioactive waste repository, which mainly stores radioactive material scrapped by Shanghai. The waste repository is located far from the urban area, and the staff at the repository has rich experience in radioactive source utility and protection. The experimental site was selected as a rectangular area of $30 \times 25 \mathrm{~m}$ in the repository site. To ensure the safety of the $\mathrm{UAV}$, the vegetation in the area consisted mostly of shrubs and no tall trees. The UAV platform used in the experiments was the quadrotor DJI Matrice 100 (M100) instead of the M600, considering the size and topography of the experimental site. Despite the difference in mechanical architecture, the API of the M100 is generally the same, which means that the same search program as the M600 was used in the experiments. Four ${ }^{241}$ Am radioactive sources of radioactivity were selected within $3.7 \times 10^{9} \mathrm{~Bq}$, which has a half-life of 432 years in a lead canister and can be considered as one radioactive source. The manufacture date of this device is 9 December 2005. As measured, the dose on the surface of the radioactive source was $100 \mu \mathrm{Sv}$. Due to the low activity of the radioactive source, the second and third radioactive source search algorithms were not usable, because the monitoring data are not sufficiently significant when the UAV is not located adequately close to the source. Therefore, this experiment focuses on verifying the effect of the first radioactive source search algorithm.

Since the frequency of the radiation dose rate returned by the radiation detection sensor is $1 \mathrm{~Hz}$, the slower the cruising speed of the UAV is, the higher the search accuracy that can be obtained. Therefore, in order to improve the search accuracy, the UAV's cruising speed should be reduced as much as possible under the premise of effective flight time. Unlike the M600, the effective flight time of 
the M100 UAV equipped with an uncontrolled radioactive source search system is only about $15 \mathrm{~min}$. Considering the size of the search area and in order to achieve a satisfactory level of accuracy, the step size in search of the short side was set to $1 \mathrm{~m}$, the cruising speed was set to $1 \mathrm{~m} / \mathrm{s}$, and the flight altitude was set to $10 \mathrm{~m}$. The initial position of the UAV in the southwest corner of the region and all of the collected dose rate information was recorded in the document with the corresponding position information. After planning the route and creating the search plan, the experiment was divided into two components. The first flight was conducted in the absence of a radioactive source in the area. This flight was used to observe the drift of the sensor and verify the stability of the sensor. The staff subsequently picked out the radioactive source and placed it randomly at any location within the area, and the second flight was conducted to search for the radioactive source. This flight was mainly used to verify the effect of the radioactive source search algorithm. After the flight, the data were exported, and MATLAB was used to interpolate the data and plot the dose rate distribution in the search area. It only took $3 \mathrm{~min}$ to interpolate and plot the radiation distribution nephogram, which fully meets the real-time data processing requirements of uncontrolled radioactive source search. The interpolation results of the two flights are shown in Figure 4.

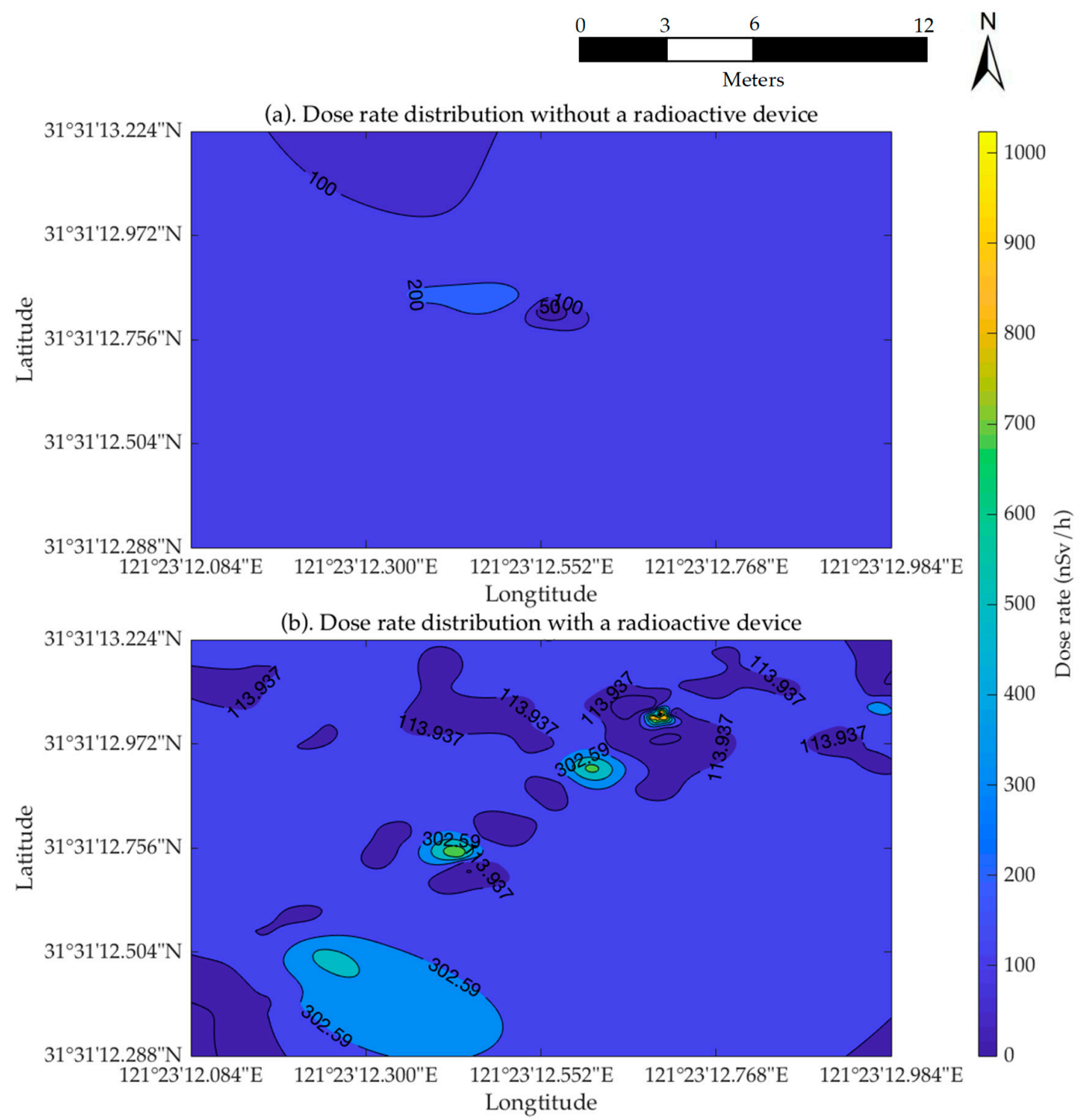

Figure 4. Dose rate distribution in experiments. (a) Dose rate distribution without a radioactive source; (b) dose rate distribution with a radioactive source. 


\section{Results}

As shown in Figure 4a, the flight interpolation results are calculated using a UAV controlled by the source search algorithm when no radiation is present in the search area. As shown in the figure, the dose rate in the area monitored by the sensor changes minimally due to the absence of a radioactive source. The minimum monitoring value of the sensor is $123 \mathrm{nSv} / \mathrm{h}$, the maximum value is $235 \mathrm{nSv} / \mathrm{h}$, the average value is $132 \mathrm{nSv} / \mathrm{h}$, and the standard deviation of the monitoring data is $15 \mathrm{nSv} / \mathrm{h}$. It can be observed that the stability of the sensor is high, and no drastic data drift occurs during the measurement. Figure $4 \mathrm{~b}$ shows the dose rate distribution of the region measured by the UAV after the radioactive source was randomly placed in the search area. It can be observed from the figure that the dose rate distribution within the region changed significantly due to the addition of the radioactive sources. The location of the radioactive source can be determined by finding the highest point of the dose rate in the region. Via analysis of the data, it was found that the highest dose rate in the region was $1023 \mathrm{nSv} / \mathrm{h}$ at the point $\left(121^{\circ} 23^{\prime} 12.69^{\prime \prime}, 31^{\circ} 31^{\prime} 13.04^{\prime \prime}\right)$.

In a specific field experiment, after the UAV has obtained the distribution of the dose rate in the entire region, the position of the radioactive source is determined by confirming the position of the highest dose rate. In the entire experiment, the battery performance of the UAV platform was good and met the requirement of 15 min' effective flight time in the study. In the coordinate system in which the take-off point is the origin, the $x$-axis and $y$-axis point to the north and the east, respectively. The coordinate of the point is $(15.1,14.3)$, and the coordinate of the radioactive source placed by the staff was $(15,14)$. The error between the two positions is $0.32 \mathrm{~m}$, which satisfies the accuracy requirements for emergent search and treatment. However, the radioactive source was placed in a lead canister, and only the top of the canister was opened in the experiment, so a strong dose rate can be obtained only from above the lead canister. That is also why Figure $4 \mathrm{~b}$ does not show a gradient distribution of the dose rate. The presence of certain anomalies can be noted in the figure, and these anomalies are mainly manifested in a sudden increase in the dose rate measured at the point where no radioactive source occurs. This is likely due to a measurement drift from the sensor in a strong radiation environment. However, it is worth noting that the dose rates at these three sites do not exceed the dose rate measured at the top of the radioactive source. Thus, the measurement drift of the sensor does not affect the final source search result.

\section{Discussion}

From Figure 4, we can see that some values drifted in the specific measurement by the radiation detection sensor. Since the data drift is not large, there is no effect on the traversal search algorithm, which takes all the dose rate values in the region into account. However, there are few monitoring values available for the binary and successive approximation algorithms, so any tiny fluctuations of the sensor may make the algorithms make erroneous judgments and eventually lead to the failure of the radioactive source search. Due to the safety consideration, as well as the limitation of relevant policies and regulations, the activity of the radioactive source in the experiment was relatively low and was also placed in a lead canister to avoid excessive radiation. The dose rate near the boundary of the region was almost close to the background value and did not meet the requirements of the latter two algorithms. As a result, we did not evaluate the two algorithms in the current study. To better evaluate the performance of the UAV-based radioactive source search platform compared to other platforms, we also need to conduct experiments among multiple platforms, for example, a ground vehicle combining GNSS and the radiation detector. However, the radioactive source search vehicle of the Shanghai Institute for Supervision over Radio-environment did not have GNSS support and therefore could not generate the radiation distribution map for the UAV platform. Limited by these factors, it is still difficult for us to do comparison among platforms for the spatial search at present.

As shown in Figure 4, the radioactive source search system based on the first search algorithm is able to locate the radioactive source within a region by detecting the dose rate distribution within the region. Compared with the large-crowd strategy and radiation detection vehicles, the search system 
based on a UAV can achieve detection and location of a radioactive source in a region without the risk of requiring emergency personnel to enter the region. This strategy ensures the safety of personnel and improves the efficiency of the search activity. This search system can perform high-altitude, high-speed flight to generate a radiation distribution map of a large area, similar to fixed-wing UAVs, but can also conduct low-altitude, low-speed flight to detect a low-activity/small-volume radioactive source, which is the critical point of difficulty in the search for uncontrolled radioactive sources. Benefitting from the flight characteristics of low-altitude/low-speed multi-rotor UAVs, the proposed search system compensates for the shortcomings of the conventional fixed-wing UAVs for radioactive source searches. The system can search for uncontrolled radioactive sources with lower activity (classified as IV or below). After the discovery of the radioactive source, the system can also perform subsequent processing, such as the capture of the radioactive source. Furthermore, a simultaneous search for multiple radioactive sources in a region can also be achieved by the system.

\section{Conclusions}

In this study, a prototype of the UAV-based radioactive source search platform is proposed and tested. The multi-rotor UAV is modified based on a DJI Matrice 600 unit. The effective lifetime in the air of the modified UAV reaches $30 \mathrm{~min}$, and the maximum velocity in the horizontal direction is $15 \mathrm{~m} / \mathrm{s}$. Different from previous research, the platform compensates for the inability of fixed-wing UAVs to search for low-activity radioactive sources. The UAV can automatically search for an uncontrolled radioactive source in a region using a radiation sensor and a main controller at a low speed and a low altitude. Moreover, the requirements for take-off and landing sites are relatively low, which is suitable for all terrain operations, such as mountainous areas, urban areas, etc. To realize automatic detection of the uncontrolled radioactive source, three search and routing algorithms were introduced and analyzed in this study. These algorithms dictate the flying routes of the UAV using three different approaches with the same objective of locating the uncontrolled radioactive source in the shortest time. A field experiment was performed in this study to test the effect of the first algorithm. The experimental results show that the algorithm can accurately find the radioactive source in the region. The real-time transmission of video between the air and the ground also offers additional terrain information for the region, which could also aid in preventing collisions by taking the corresponding actions. Once the uncontrolled radioactive source has been located, the video transmission can also supply information on the terrain near the radioactive source, which could facilitate rapid and safe treatment of the source.

In follow-up studies, we expect to improve the sensor in order to obtain a more stable measurement reading and consider using high activity radioactive sources taking out the lead canister in the experiment. Then, we can complete the performance evaluation of all the algorithms. We will look for a ground vehicle combining GNSS with the radiation detector and larger sites for comparison experiments. The resolution and efficiency of this system will also be enhanced, and a manipulator will be installed on the UAV to realize an automatic search and controlled grab of radioactive sources. In the future, a search scenario with multiple radioactive sources in a region will also be included in the discussion. Furthermore, to search over larger regions, cooperative radioactive source search via multi-rotor UAVs will be investigated in future work.

\section{Patents}

The authors declare a patent for utility models based on the search system applied by the State Intellectual Property Office of the People's Republic of China. The patent applicants are Shanghai Jiao Tong University and Shanghai Station of Radiation Environment Supervision. The inventors are Zhong-Ren Peng, Bai Li, and Lixin Ge. The application number is 201721012785.X. We have received a grant of patent for the invention. The specific aspect of manuscript covered in patent application is in the Materials and Methods section. 
Author Contributions: B.L. wrote the main manuscript text, implemented the UAV-based radioactive source search system, and analyzed the data. Z.-R.P. provided the original research ideas and framework. B.L., Z.-R.P., Z.W., and L.G. conceived and designed the field experiment. Z.-R.P., Y.Z., Z.W., and C.L. edited this manuscript. Z.-R.P., Z.W., and L.G. are the PIs of respective grant that provided funding and supervised the study, and have carefully edited the paper. All authors reviewed the manuscript.

Acknowledgments: This work was supported by National Key R\&D Program of China (2016YFC0200500), the National Natural Science Foundation of China (No. 41701552), the Science and Technology Project of Guangzhou, China (No. 201803030032), the State Key Laboratory of Ocean Engineering at Shanghai Jiao Tong University (GKZD010071), and the Shanghai Environmental Protection Bureau (No. 2015-7). The authors thank the Shanghai Station of Radiation Environment Supervision for supplying technical support and professional suggestions.

Conflicts of Interest: The authors declare no conflict of interest. The founding sponsors had no role in the design of the study; in the collection, analyses, or interpretation of data; in the writing of the manuscript; or in the decision to publish the results.

\section{References}

1. Aage, H.K.; Korsbech, U. Search for lost or orphan radioactive sources based on NaI gamma spectrometry. Appl. Radiat. Isot. 2003, 58, 103-113. [CrossRef]

2. Cresswell, A.J.; Sanderson, D.C.W. The use of difference spectra with a filtered rolling average background in mobile gamma spectrometry measurements. Nucl. Instrum. Methods Phys. Res. Sect. A Accel. Spectrom. Detect. Assoc. Equip. 2009, 607, 685-694. [CrossRef]

3. Qipu, Z.; Chen, D.; Xiaojian, Z.; Wang, X. Emergency Investigation and Analysis of the Radiation Accident of Nanjing 192Ir Radioactive Source. Chin. J. Radiol. Med. Prot. 2014, 8, 561-562.

4. Kock, P.; Finck, R.R.; Nilsson, J.M.C.; Östlund, K.; Samuelsson, C. A deviation display method for visualising data in mobile gamma-ray spectrometry. Appl. Radiat. Isot. 2010, 68, 1832-1838. [CrossRef] [PubMed]

5. Kock, P.; Rääf, C.; Samuelsson, C. On background radiation gradients-The use of airborne surveys when searching for orphan sources using mobile gamma-ray spectrometry. J. Environ. Radioact. 2014, 128, 84-90. [CrossRef] [PubMed]

6. Kock, P.; Lanke, J.; Samuelsson, C. A real-time statistical alarm method for mobile gamma spectrometry-Combining counts of pulses with spectral distribution of pulses. Nucl. Instrum. Methods Phys. Res. Sect. A Accel. Spectrom. Detect. Assoc. Equip. 2012, 681, 55-60. [CrossRef]

7. Stephens, D.L.; Runkle, R.C.; Carlson, D.K.; Peurrung, A.J.; Seifert, A.; Wyatt, C. Induced temporal signatures for point-source detection. IEEE Trans. Nucl. Sci. 2005, 52, 1712-1715. [CrossRef]

8. Ziock, K.P.; Collins, J.W.; Fabris, L.; Gallagher, S.; Horn, B.K.P.; Lanza, R.C.; Madden, N.W. Source-search sensitivity of a large-area, coded-aperture, gamma-ray imager. IEEE Trans. Nucl. Sci. 2006, 53, 1614-1621. [CrossRef]

9. Břennan, S.M.; Mielke, A.M.; Torney, D.C.; Brennan, S.M.; Mielke, A.M.; Torney, D.C. Radioactive source detection by sensor networks. IEEE Trans. Nucl. Sci. 2005, 52, 813-819. [CrossRef]

10. Stephens, J.D.L.; Peurrung, A.J. Detection of moving radioactive sources using sensor networks. IEEE Trans. Nucl. Sci. 2004, 51, 2273-2278. [CrossRef]

11. Nemzek, R.J.; Dreicer, J.S.; Torney, D.C.; Warnock, T.T. Distributed sensor networks for detection of mobile radioactive sources. IEEE Trans. Nucl. Sci. 2004, 51, 1693-1700. [CrossRef]

12. Brennan, S.M.; Mielke, A.M.; Torney, D.C.; Maccabe, A.B. Radiation detection with distributed sensor networks. Computer 2004, 37, 57-59. [CrossRef]

13. Brady, J.M.; Stokes, M.D.; Bonnardel, J.; Bertram, T.H. Characterization of a Quadrotor Unmanned Aircraft System for Aerosol-Particle-Concentration Measurements. Environ. Sci. Technol. 2016, 50, 1376-1383. [CrossRef] [PubMed]

14. Šmídl, V.; Hofman, R. Tracking of atmospheric release of pollution using unmanned aerial vehicles. Atmos. Environ. 2013. [CrossRef]

15. Pöllänen, R.; Toivonen, H.; Peräjärvi, K.; Karhunen, T.; Smolander, P.; Ilander, T.; Rintala, K.; Katajainen, T.; Niemelä, J.; Juusela, M.; et al. Performance of an air sampler and a gamma-ray detector in a small unmanned aerial vehicle. J. Radioanal. Nucl. Chem. 2009, 282, 433-437. [CrossRef]

16. Zhu, S.; Wang, D.; Low, C.B. Cooperative control of multiple UAVs for source seeking. J. Intell. Robot. Syst. Theory Appl. 2013, 70, 293-301. [CrossRef] 
17. Han, J.; Di, L.; Coopmans, C.; Chen, Y. Fractional Order Controller for Pitch Loop Control of a VTOL UAV. Int. Conf. Unmanned Aircr. Syst. 2013, 609-614. [CrossRef]

18. Chao, H.; Luo, Y.; Di, L.; Chen, Y.Q. Roll-channel fractional order controller design for a small fixed-wing unmanned aerial vehicle. Control Eng. Pract. 2010, 18, 761-772. [CrossRef]

19. Di, L.; Chao, H.; Han, J.; Chen, Y. Cognitive Multi-UAV Formation Flight: Principle, Low-Cost UAV Testbed, Controller Tuning and Experiments. In Proceedings of the ASME 2011 International Design Engineering Technical Conferences and Computers and Information in Engineering Conference, Washington, DC, USA, 28-31 August 2011; pp. 917-927.

20. Han, J.; Xu, Y.; Di, L.; Chen, Y. Low-cost multi-UAV technologies for contour mapping of nuclear radiation field. J. Intell. Robot. Syst. Theory Appl. 2013, 70, 401-410. [CrossRef]

21. Han, J.; Chen, Y. Multiple UAV formations for cooperative source seeking and contour mapping of a radiative signal field. J. Intell. Robot. Syst. Theory Appl. 2014, 74, 323-332. [CrossRef]

(C) 2018 by the authors. Licensee MDPI, Basel, Switzerland. This article is an open access article distributed under the terms and conditions of the Creative Commons Attribution (CC BY) license (http:/ / creativecommons.org/licenses/by/4.0/). 